\title{
Separation of Alkyne Enantiomers by Chiral Column HPLC Analysis of Their Cobalt-Complexes
}

\author{
Qiaoyun Liu ${ }^{1}$, Jing Wang ${ }^{1,2}$, Junfei $\mathrm{Li}^{1}{ }^{1}$, Xiaolei Wang ${ }^{2}$, Shichao $\mathrm{Lu}^{2}$, Xuan $\mathrm{Li}^{2}$, Yaling Gong ${ }^{2, *}$ \\ and $\mathrm{Shu} \mathrm{Xu}^{2, *}$ \\ 1 School of Chemistry and Material Science, Shanxi Normal University, 1 Gongyuan Street, Linfen, \\ Shanxi 041004, China; liuqy@sxnu.edu.cn (Q.L.); 214111062@stu.sxnu.edu.cn (J.W.); \\ lijunfei@sxnu.edu.cn (J.L.) \\ 2 State Key Laboratory of Bioactive Substance and Function of Natural Medicines, Beijing Key Laboratory of \\ Active Substances Discovery and Drugability Evaluation, Institute of Materia Medica, Chinese Academy of \\ Medical Sciences and Peking Union Medical College, 2A NanWei Road, Xicheng Distrct, \\ Beijing 100050, China; wangxiaolei@imm.ac.cn (X.W.); lushichao@imm.ac.cn (S.L.); \\ lanthanum1979@gmail.com (X.L.) \\ * $\quad$ Correspondence: ylgong@imm.ac.cn (Y.G.); xushu@imm.ac.cn (S.X.); Tel.: +86-10-6316-5243 (S.X.)
}

Academic Editor: Arnaud Gautier

Received: 20 January 2017; Accepted: 11 March 2017; Published: 20 March 2017

\begin{abstract}
Separation of the enantiomers of new chiral alkynes in strategic syntheses and bioorthogonal studies is always problematic. The chiral column high-performance liquid chromatography (HPLC) method in general could not be directly used to resolve such substrates, since the differentiation of the alkyne segment with the other alkane/alkene segment is not significant in the stationary phase, and the alkyne group is not a good UV chromophore. Usually, a pre-column derivatization reaction with a tedious workup procedure is needed. Making use of easily-prepared stable alkyne-cobalt-complexes, we developed a simple and general method by analyzing the in situ generated cobalt-complex of chiral alkynes using chiral column HPLC. This new method is especially suitable for the alkynes without chromophores and other derivable groups.
\end{abstract}

Keywords: alkyne; cobalt complex; HPLC; chiral column; resolution

\section{Introduction}

Alkyne is one of the fundamental groups in organic chemistry, which exists widely in natural products and unnatural functionalized molecules [1]. The development of modern transition-metal-catalyzed reactions, such as cross coupling [2], Pauson-Khand reaction [3], click chemistry [4], etc., has caused alkynes to play a more and more important role in strategic syntheses and bioorthogonal design [1].

Enantiomeric purity is vital for chiral alkynes to function. The enantiomeric excess (ee) of chiral alkynes with unknown optical rotation could be determined generally in two ways: (1) using another chiral reagent to derivate diastereomers and then measure the diasteromeric ratio; (2) using enantioselective chromatography to directly measure the enantiomeric excess. Recently, in the course of our total synthesis of natural products, we prepared the known alkyne 1a (Figure 1), and found that two methods were used in the literatures to determine its ee [5-13]. Most literature used Mosher's methodology [14] which undertook an esterification with expensive Mosher's chiral reagent, and determined the enantiomeric purity by NMR analysis of the resultant diasteromeric mixture [5-12]. One study used high-performance liquid chromatography (HPLC) with chiral column to analyze the 3,5-dinitrobenzoate derivative of 1a [13]. Since both methods need a derivatization reaction with tedious workup procedure, we initially attempted a direct analysis of 1a with chiral column HPLC 
method. However, no good result was achieved, which may be due to two causes: (1) the alkyneand alkene-chains in 1a are similar in size, and therefore might be difficult to be differentiated by chiral stationary phase. In fact, no enantiomeric separation could be found in all our attempted chiral columns and HPLC conditions; (2) alkyne and other functional groups in 1a are all poor chromophores. As a result, 1a showed very low UV-absorption, and it was difficult to characterize under normal HPLC concentration using a UV detector.



Figure 1. Preparation of 2a and the comparison of its properties with those of 1a. (a) Transformation from 1a to 2a; (b) The UV-Vis spectra of $1 \mathrm{mM}$ solution of 1a in $n$-hexane; (c) The UV-Vis spectra of $0.01 \mathrm{mM}$ solution of 2a in $n$-hexane; (d) The high-performance liquid chromatography (HPLC) chromatogram of injected 1a (20 $\mu \mathrm{L}, 1 \mathrm{M}$ in $n$-hexane), with CHIRALPAK-IB column, 2 -PrOH/ $n$-hexane 0.4:99.6 eluting-solvent system, $1 \mathrm{~mL} \cdot \mathrm{min}^{-1}$ flow rate, and $200 \mathrm{~nm}$ detection wavelength at $25^{\circ} \mathrm{C}$; (e) The HPLC chromatogram of racemic $2 \mathbf{a}(20 \mu \mathrm{L}, 1 \mathrm{mM}$ in $n$-hexane) with the same conditions as (d) except the detection wavelength of $350 \mathrm{~nm}$; (f) The HPLC chart of enantioenriched 2a with the same conditions as (e). 
Aware of the insufficiency of the present ee determination methodology for chiral alkynes such as 1a, we decided to develop and report herein a simple and general method by analyzing the in situ generated cobalt-complex of chiral alkynes using chiral column HPLC. This new method is especially suitable for the alkynes without chromophores and other derivable groups.

\section{Results and Discussion}

To overcome the above-mentioned two weak points of alkynes, our idea was to make use of its metal complex. It is well known that $\mathrm{Co}_{2}(\mathrm{CO})_{8}$ can easily react with alkynes to lose two carbon monoxides and form stable $\mathrm{Co}_{2}(\mathrm{CO})_{6}$-alkyne complexes. Figure 1a showed this transformation from alkyne $\mathbf{1 a}$ to its complex 2a. By this simple complexation, (1) the alkyne-chain became significantly bulky, which was very different in size from the other side-chain; (2) the Co-complexation substructure was a good chromophore, making the UV absorption of 2a more than 100 times stronger than 1a (Figure $1 \mathrm{~b}, \mathrm{c})$, and a wide range of detection wavelength $(200-400 \mathrm{~nm})$ could be selected in the HPLC experiment. As a result, comparison with the unsuccessful result of 1a (Figure 1d), 2a was easily resolved by HPLC using a CHIRALPAK-IB column with 0.4:99.6 2-PrOH/n-hexane eluting-solvent system, $1 \mathrm{~mL} \cdot \mathrm{min}^{-1}$ flow rate, and $350 \mathrm{~nm}$ detection wavelength at $25^{\circ} \mathrm{C}$ (Figure 1e). Under the same conditions, an enantioenriched 2a was also tested (Figure 1f), and the detected enantiomeric ratio was consistent with that determined by the modified Mosher's methodology [11].

After confirmation of our idea on compounds 1a and 2a, we next prepared a series of $\mathrm{Co}_{2}(\mathrm{CO})_{6}$-alkyne complexes $\mathbf{2} \mathbf{b}-\mathbf{k}$ (Table 1 ) from none-chromophore alkynes, and checked their resolution by HPLC chiral column. As a result, all the enantiomeric pairs of Co-complexes were successfully baseline-separated. For alcohol substrates, the size of another side-chain (butyl, allyl, ethyl, methyl) or the distance between the $\mathrm{C} \equiv \mathrm{C}$ triple bond and the hydroxyl group did not show any notable effect for the enantiomeric separation ( $2 \mathbf{a}-\mathbf{e})$. Even a tertiary alkynol-complex $2 \mathbf{f}$ could be well resolved, although a CHIRALPAK-IA column was used in this case. Internal alkyne-complexes $\mathbf{2} \mathbf{g}$ and $\mathbf{2 h}$ also gave good separation. Replacing the hydroxyl group with halogen atoms might change the interaction between the substrates and the stationary phase of the column. Fortunately, the chloride $2 \mathbf{i}$ and the fluoride $\mathbf{2} \mathbf{j}$ were both resolved, although lower polar eluting-solvent systems were needed for the low polarity of these substrates. Notably, the tetrahydropyran (THP)-protected alkynol-complex 2k could also been enantiomerically separated. Actually, compounds such as $\mathbf{1} \mathbf{i}-\mathbf{k}$, are not good substrates for classical pre-column derivatization methods.

Table 1. Scope of alkynes for HPLC resolution of their Co-complexes.

Alkyne (1)


Table 1. Cont.

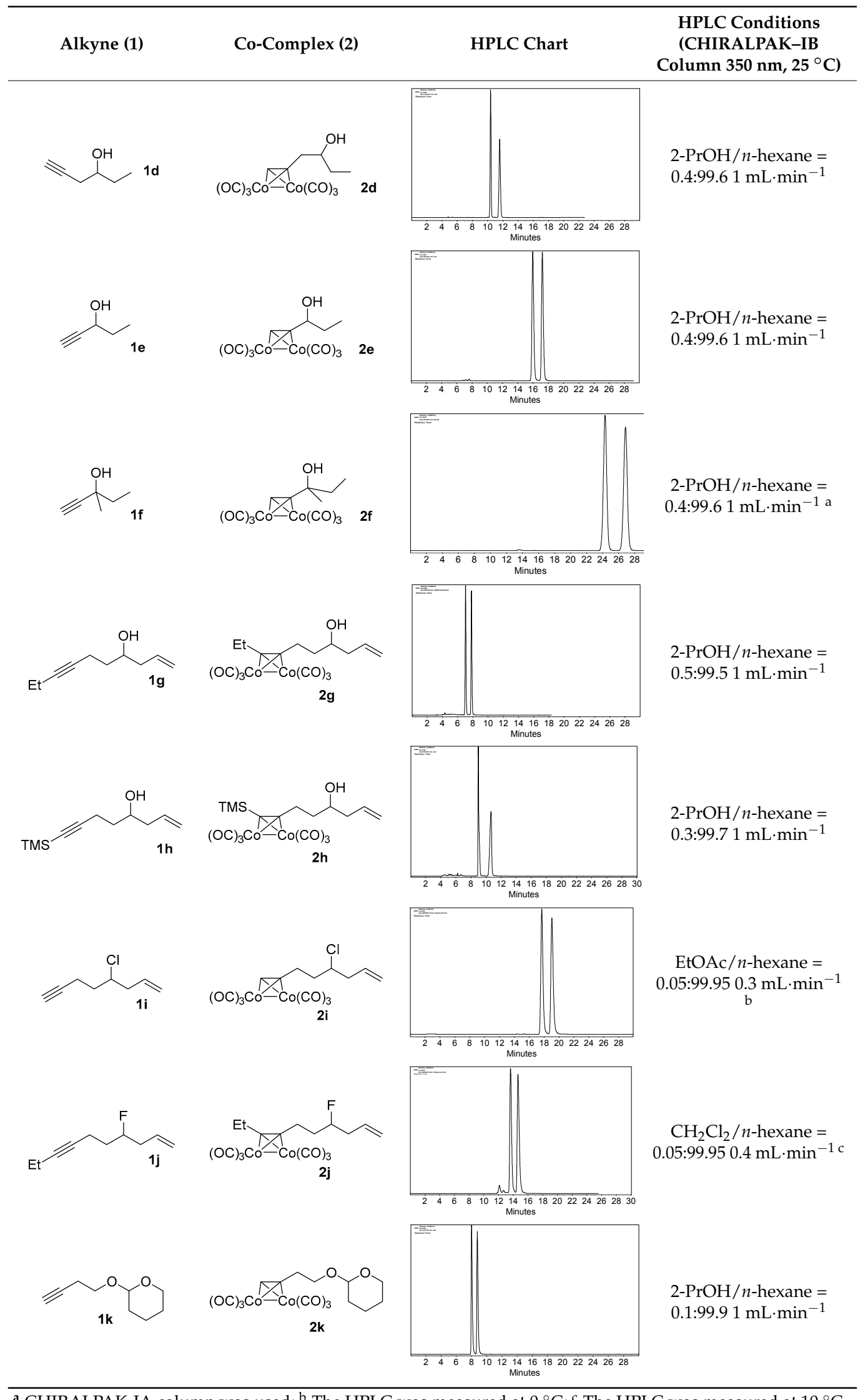

${ }^{\text {a }}$ CHIRALPAK-IA column was used; ${ }^{b}$ The HPLC was measured at $0{ }^{\circ} \mathrm{C} ;{ }^{\mathrm{c}}$ The HPLC was measured at $10^{\circ} \mathrm{C}$. 
It was noted that most of the above HPLC experiments were carried out with the same chiral column using the most general 2-PrOH/n-hexane eluting-solvent system. Except for changing the 2-PrOH $/ n$-hexane ratio to make the retention time between 10-20 min, no more optimization was done for HPLC conditions. The simple setup of the HPLC conditions implied the easiness and generality of our Co-complex analytic method on much wider alkyne substrates.

Although the above HPLC analysis was all carried out with purified Co-complexes, since the CHIRALPAK immobilized-polysaccharide type columns can tolerate a wide range of solvents, we next attempted to directly measure the reaction system of $\mathbf{1 a}, \mathrm{Co}_{2}(\mathrm{CO})_{8}$, and $\mathrm{CH}_{2} \mathrm{Cl}_{2}$ (Figure $1 \mathrm{a}$ ). To our delight, by just diluting a small amount of the reaction mixture with $n$-hexane and then injection into HPLC, the in situ generated 2 a could be monitored without any problems (Figure 2). Since the manipulation was rather simple without the tedious workup procedure like those of Mosher's or other carbonyl chloride methods [5-13], this result implied a promising application of our Co-complexation method in monitoring the asymmetric alkyne-synthesis reactions.

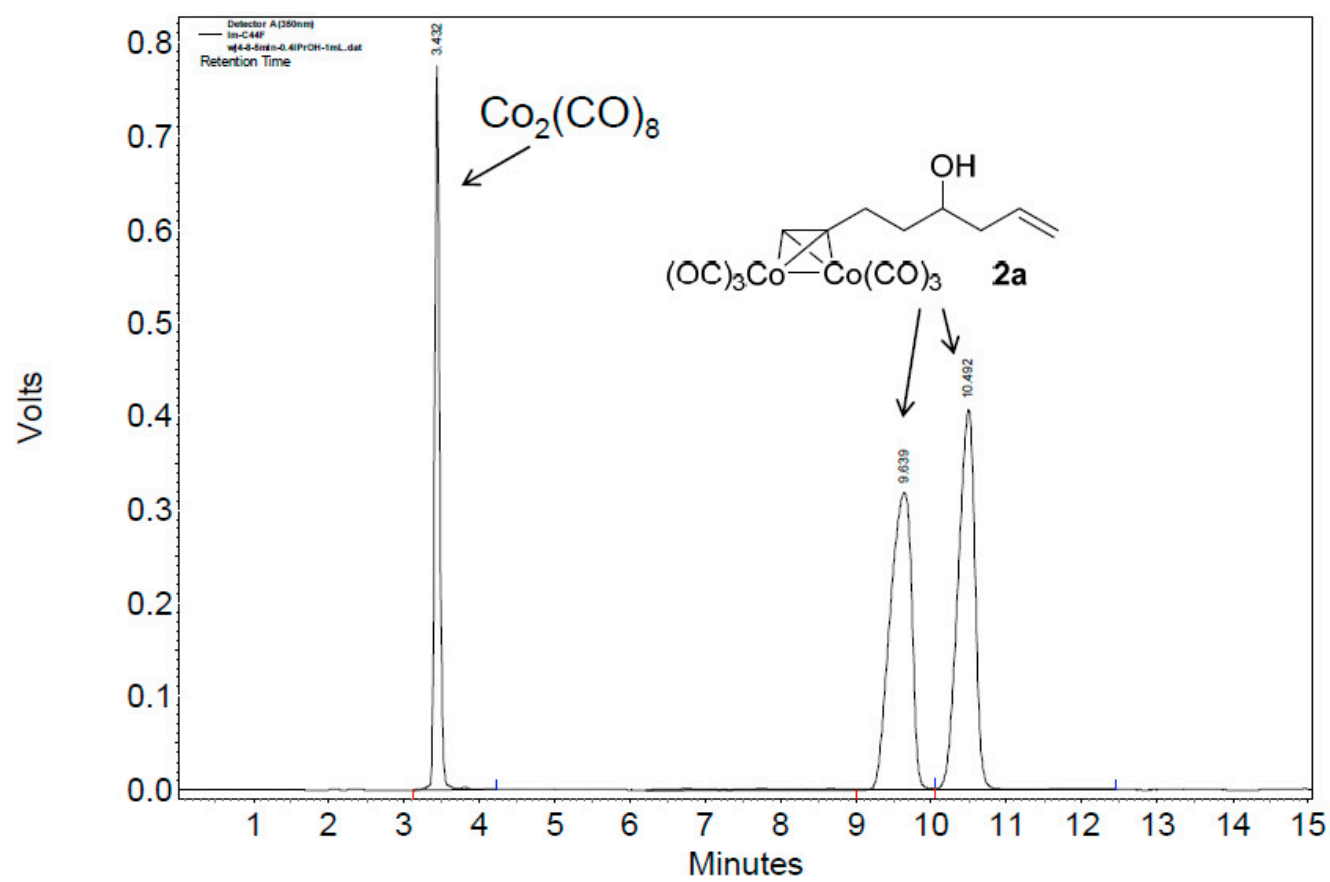

Figure 2. The HPLC chromatogram of the reaction mixture of $1 \mathrm{a}, \mathrm{Co}_{2}(\mathrm{CO})_{8}$, and $\mathrm{CH}_{2} \mathrm{Cl}_{2}$, with CHIRALPAK-IB column, 2-PrOH/n-hexane 0.4:99.6, $1 \mathrm{~mL} \cdot \mathrm{min}^{-1}$ flow rate, and $350 \mathrm{~nm}$ detection wavelength at $25{ }^{\circ} \mathrm{C}$. Sample preparation: $20 \mu \mathrm{L}$ of the reaction mixture was taken 5 min after the reaction started, diluted with $0.5 \mathrm{~mL} n$-hexane, and passed through a disposable syringe filter (Nylon 66, $0.22 \mu \mathrm{m}, 13 \mathrm{~mm}) ; 5 \mu \mathrm{L}$ of the filtration was injected.

\section{Materials and Methods}

\subsection{General Methods}

All reactions were carried out under an atmosphere of Ar unless otherwise indicated. ${ }^{1} \mathrm{H}-$, and ${ }^{13}$ C-NMR spectra were acquired on Mercury-300 (Agilent, Santa Clara, CA, USA), AVANCE III-400 (Bruker, Billerica, MA, USA), WNMR-I-500 (Zhongke Niujin Co., Ltd., Wuhan, China), or VNMRS-600 spectrometers (Agilent). Chemical shifts are indicated in parts per million (ppm) downfield from tetramethylsilane (TMS, $\delta=0.00$ ) with residual undeuterated solvent peaks as internal reference for ${ }^{1} \mathrm{H}-\mathrm{NMR}$ and deuterated solvent peaks shifts for ${ }^{13} \mathrm{C}-\mathrm{NMR}$. Multiplicities are reported as s (singlet), $\mathrm{d}$ (doublet), $\mathrm{t}$ (triplet), q (quartet), m (multiplet), br (broad) or combinations of those. For NMR analysis of the $\mathrm{Co}_{2}(\mathrm{CO})_{6}$-alkyne complexes, the sample solution in $\mathrm{CDCl}_{3}$ should pass through a 
disposable syringe filter (Nylon 66, $0.22 \mu \mathrm{m}, 13 \mathrm{~mm}$ ) immediately before the NMR experiment, to remove the small amount of paramagnetic material. Mass spectra (MS) are electron ionization (EI) or electrospray ionization (ESI). EI-MS data were measured on GCT mass spectrometer (Micromass, Manchester, UK). ESI-MS data were measured on Thermo-Fisher Accela liquid chromatography system coupled with Exactive Plus Orbitrap mass spectrometer (Thermo-Fisher, Bremen, Germany). Reagents and compounds $\mathbf{1} \mathbf{c}-\mathbf{f}, \mathbf{1} \mathbf{k}$ were purchased from commercial suppliers and used as received. Compound $\mathbf{1 a}$ was prepared according to the literature procedure [8]. Compounds $\mathbf{1} \mathbf{b}, \mathbf{1} \mathbf{g}-\mathbf{j}$, and $\mathbf{2} \mathbf{a}-\mathbf{k}$ were synthesized as following. See Supplementary Materials for spectra and chromatograms of the prepared products.

\subsection{Preparation of Alkynes and Compound Characterization}

Non-1-yn-5-ol (1b). To dry THF (254 mL) was added $n$-BuLi (2.5 M in $n$-hexane, $9.74 \mathrm{~mL}, 24.4 \mathrm{mmol})$ at $-78^{\circ} \mathrm{C}$. 4-pentyn-1-al [8] $(1.00 \mathrm{~g}, 12.2 \mathrm{mmol})$ was added slowly at $-78^{\circ} \mathrm{C}$. Then the mixture was stirred for $3 \mathrm{~h}$ while its temperature reached $23^{\circ} \mathrm{C}$. Methanol $(50 \mathrm{~mL})$ was added to quench excess $n$-BuLi. The mixture was concentrated. The residue was dissolved in $10 \%$ aqueous $\mathrm{HCl}$ and the resulting mixture was extracted with $\mathrm{CH}_{2} \mathrm{Cl}_{2}$. The combined organic layers were dried over $\mathrm{Na}_{2} \mathrm{SO}_{4}$, concentrated, and purified by silica gel column chromatography. Elution with cyclohexane/acetone (50/1) gave a pale yellow oil $(0.38 \mathrm{~g}, 22 \%)$; ${ }^{1} \mathrm{H}-\mathrm{NMR}\left(300 \mathrm{MHz}, \mathrm{CDCl}_{3}\right) \delta 3.75(\mathrm{br}, 1 \mathrm{H}, \mathrm{CHOH}), 2.34(\mathrm{t}, J=6.0 \mathrm{~Hz}, 2 \mathrm{H})$, $1.98(\mathrm{~s}, 1 \mathrm{H}, \equiv \mathrm{CH}), 1.72-1.60(\mathrm{~m}, 2 \mathrm{H}), 1.67(\mathrm{br}, 1 \mathrm{H}, \mathrm{OH}), 1.46-1.33(\mathrm{~m}, 6 \mathrm{H}), 0.91\left(\mathrm{t}, J=6.3 \mathrm{~Hz}, 3 \mathrm{H}, \mathrm{CH}_{3}\right)$. ${ }^{13} \mathrm{C}-\mathrm{NMR}\left(75 \mathrm{MHz}, \mathrm{CDCl}_{3}\right) \delta 84.5(\mathrm{C} \equiv \mathrm{CH}), 71.0,68.9,37.3,35.9,28.0,22.9,15.2,14.3$. HRMS (ESI) $\mathrm{m} / \mathrm{z}$ calcd. for $\mathrm{C}_{9} \mathrm{H}_{17} \mathrm{O}^{+}[\mathrm{M}+\mathrm{H}]^{+}$: 141.1274; found: 141.1269 .

Dec-1-en-7-yn-4-ol (19). To a solution of tert-butyldimethyl(oct-1-en-7-yn-4-yloxy)silane [8] (3.00 g, $12.6 \mathrm{mmol})$ in dry THF $(15 \mathrm{~mL})$ cooled at $-78^{\circ} \mathrm{C}$ was added dropwise lithium diisopropylamide (LDA, $10.064 \mathrm{~mL}, 2.5 \mathrm{M}$ in THF, $25.16 \mathrm{mmol}$ ) via syringe. The resulting mixture was stirred at $-78^{\circ} \mathrm{C}$ for $1 \mathrm{~h}$ followed by addition of iodoethane $(5.056 \mathrm{~mol}, 62.9 \mathrm{mmol})$. The reaction solution allowed to warm to room temperature. After being stirred for $26 \mathrm{~h}$, the mixture was passed through a silica-gel pad (eluted with $\mathrm{CH}_{2} \mathrm{Cl}_{2}$ ), and concentrated in vacuum. To the residue was added tetrabutylammonium fluoride (1 M in THF, $39.4 \mathrm{~mL}, 39.4 \mathrm{mmol}$ ) at room temperature. The reaction mixture was stirred for $10 \mathrm{~h}$, and then was quenched with saturated aqueous $\mathrm{NH}_{4} \mathrm{Cl}$. The aqueous layer was extracted with $\mathrm{CH}_{2} \mathrm{Cl}_{2}$. The organic phase was then dried over $\mathrm{Na}_{2} \mathrm{SO}_{4}$, concentrated, and purified by silica gel column chromatography. Elution with cyclohexane $/ \mathrm{CH}_{2} \mathrm{Cl}_{2}(30 / 1)$ gave a pale yellow oil $(0.98 \mathrm{~g}$, $51 \%) ;{ }^{1} \mathrm{H}-\mathrm{NMR}\left(500 \mathrm{MHz}, \mathrm{CDCl}_{3}\right) \delta 5.82\left(\mathrm{~m}, 1 \mathrm{H}, \mathrm{CH}=\mathrm{CH}_{2}\right), 5.14-5.11\left(\mathrm{~m}, 2 \mathrm{H}, \mathrm{CH}=\mathrm{CH}_{2}\right), 3.80(\mathrm{~m}, 1 \mathrm{H}$, $\mathrm{CH}-\mathrm{OH}), 2.32-2.26(\mathrm{~m}, 3 \mathrm{H}), 2.21-2.11(\mathrm{~m}, 3 \mathrm{H}), 2.00(\mathrm{br}, 1 \mathrm{H}, \mathrm{OH}), 1.66(\mathrm{~m}, 1 \mathrm{H}), 1.60(\mathrm{~m}, 1 \mathrm{H}), 1.10$ $\left(\mathrm{t}, J=7.5 \mathrm{~Hz}, 3 \mathrm{H}, \mathrm{CH}_{3}\right) .{ }^{13} \mathrm{C}-\mathrm{NMR}\left(125 \mathrm{MHz}, \mathrm{CDCl}_{3}\right) \delta 134.6\left(\mathrm{CH}=\mathrm{CH}_{2}\right), 118.0\left(\mathrm{CH}=\mathrm{CH}_{2}\right), 82.4,78.8$, $70.0(\mathrm{CH}-\mathrm{OH}), 41.8,35.6,15.3,14.2,12.3$. HRMS (ESI) $m / z$ calcd. for $\mathrm{C}_{10} \mathrm{H}_{17} \mathrm{O}^{+}[\mathrm{M}+\mathrm{H}]^{+}: 153.1274$; found: 153.1269 .

8-(Trimethylsilyl)oct-1-en-7-yn-4-ol (1h). To a solution of 5-(trimethylsilyl)pent-4-ynal [15] (9.17 g, $59.4 \mathrm{mmol})$ in $\mathrm{CH}_{2} \mathrm{Cl}_{2}(400 \mathrm{~mL})$ cooled at $-78{ }^{\circ} \mathrm{C}$ was added dropwise allyboronic acid pinacol ester $(12.26 \mathrm{~mL}, 65.4 \mathrm{mmol})$. The resulting mixture was stirred at $-78{ }^{\circ} \mathrm{C}$ for $1 \mathrm{~h}$, and then at $0{ }^{\circ} \mathrm{C}$ for $3 \mathrm{~h}$. The reaction was quenched by addition of water, extracted with $\mathrm{CH}_{2} \mathrm{Cl}_{2}$, and washed with brine. The organic layer was dried with $\mathrm{Na}_{2} \mathrm{SO}_{4}$ and concentrated. To the residue was added $\mathrm{CH}_{2} \mathrm{Cl}_{2}$ $(100 \mathrm{~mL})$ and triethanolamine $(15 \mathrm{~mL}, 112 \mathrm{mmol})$ at room temperature. The mixture was stirred for $3 \mathrm{~h}$, then passed through a silica gel pad (eluted with $\mathrm{CH}_{2} \mathrm{Cl}_{2}$ ), concentrated, and purified by silica gel column chromatography. Elution with cyclohexane/EtOAc (100/1) gave a yellow oil $(11.13 \mathrm{~g}$, 95\%); ${ }^{1} \mathrm{H}-\mathrm{NMR}\left(400 \mathrm{MHz}, \mathrm{CDCl}_{3}\right) \delta 5.82\left(\mathrm{~m}, 1 \mathrm{H}, \mathrm{CH}=\mathrm{CH}_{2}\right), 5.17-5.12\left(\mathrm{~m}, 2 \mathrm{H}, \mathrm{CH}=\mathrm{CH}_{2}\right), 3.79(\mathrm{~m}, 1 \mathrm{H}$, $\mathrm{CH}-\mathrm{OH}), 2.38(\mathrm{t}, J=7.2 \mathrm{~Hz}, 2 \mathrm{H}), 2.30(\mathrm{~m}, 1 \mathrm{H}), 2.20(\mathrm{~m}, 1 \mathrm{H}), 1.91(\mathrm{br}, 1 \mathrm{H}, \mathrm{OH}), 1.74-1.61(\mathrm{~m}, 2 \mathrm{H}), 0.14$ $\left(\mathrm{s}, 9 \mathrm{H}, \mathrm{CH}_{3} \times 3\right) .{ }^{13} \mathrm{C}-\mathrm{NMR}\left(100 \mathrm{MHz}, \mathrm{CDCl}_{3}\right) \delta 134.5\left(\mathrm{CH}=\mathrm{CH}_{2}\right), 118.2\left(\mathrm{CH}=\mathrm{CH}_{2}\right), 106.9(\equiv \mathrm{C}-\mathrm{Si}), 85.3$ $(\mathrm{C} \equiv \mathrm{C}-\mathrm{Si}), 70.0(\mathrm{CH}-\mathrm{OH}), 41.8,35.2,16.5,0.08\left(\mathrm{SiCH}_{3} \times 3\right)$. HRMS $(\mathrm{ESI}) \mathrm{m} / z$ calcd. for $\mathrm{C}_{11} \mathrm{H}_{21} \mathrm{OSi}^{+}$ $[\mathrm{M}+\mathrm{H}]^{+}:$197.1356; found: 197.1357. 
4-Chlorooct-1-en-7-yne (1i). To a solution of oct-1-en-7-yn-4-ol (1a, $300 \mathrm{mg}, 2.4 \mathrm{mmol})$ in $\mathrm{CH}_{2} \mathrm{Cl}_{2}(18 \mathrm{~mL})$ at $0{ }^{\circ} \mathrm{C}$, pyridine $(0.384 \mathrm{~mL}, 4.8 \mathrm{mmol})$ was then added, followed by triphosgene $(356 \mathrm{mg}, 1.2 \mathrm{mmol})$ in one portion. The solution was stirred for $5 \mathrm{~min}$ and then warmed to gentle reflux. After $6 \mathrm{~h}$, the reaction mixture was poured into a separatory funnel containing $1 \mathrm{M}$ aqueous $\mathrm{HCl}(20 \mathrm{~mL})$, and the biphasic mixture was shaken vigorously. Upon separation of layers, the aqueous layer was re-extracted with $\mathrm{CH}_{2} \mathrm{Cl}_{2}$. The organic phase was dried over $\mathrm{Na}_{2} \mathrm{SO}_{4}$, concentrated, and purified by silica gel column chromatography. Elution with cyclohexane gave a pale yellow oil (213.6 mg, 62\%); ${ }^{1} \mathrm{H}-\mathrm{NMR}(300 \mathrm{MHz}$, $\left.\mathrm{CDCl}_{3}\right) \delta 5.85\left(\mathrm{~m}, 1 \mathrm{H}, \mathrm{CH}=\mathrm{CH}_{2}\right), 5.18-5.13\left(\mathrm{~m}, 2 \mathrm{H}, \mathrm{CH}=\mathrm{CH}_{2}\right), 4.10(\mathrm{~m}, 1 \mathrm{H}, \mathrm{CH}-\mathrm{Cl}), 2.56(\mathrm{~m}, 2 \mathrm{H}), 2.41$ $(\mathrm{m}, 2 \mathrm{H}), 2.05-1.82(\mathrm{~m}, 2 \mathrm{H}), 1.98(\mathrm{~s}, 1 \mathrm{H}, \equiv \mathrm{CH}) .{ }^{13} \mathrm{C}-\mathrm{NMR}\left(75 \mathrm{MHz}, \mathrm{CDCl}_{3}\right) \delta 133.7\left(\mathrm{CH}=\mathrm{CH}_{2}\right), 118.2$ $\left(\mathrm{CH}=\mathrm{CH}_{2}\right), 82.8(\mathrm{C} \equiv \mathrm{CH}), 69.1(\mathrm{C} \equiv \mathrm{CH}), 60.8(\mathrm{CH}-\mathrm{Cl}), 42.5,36.2,15.8$. HRMS (ESI) $\mathrm{m} / \mathrm{z}$ calcd. for $\mathrm{C}_{8} \mathrm{H}_{12}{ }^{35} \mathrm{Cl}^{+}[\mathrm{M}+\mathrm{H}]^{+}$: 143.0622; found: 143.0618 .

4-Fluorodec-1-en-7-yne (1j). Dec-1-en-7-yn-4-ol (1g, $300 \mathrm{mg}, 1.96 \mathrm{mmol})$ was added to a previously

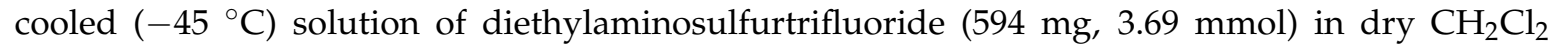
$(2.84 \mathrm{~mL})$ with vigorous stirring over a $10-\mathrm{min}$ period. The solution was allowed to come to room temperature overnight after which time it was transferred into a separatory funnel containing water and $\mathrm{CH}_{2} \mathrm{Cl}_{2}$. The organic phase was then dried over $\mathrm{Na}_{2} \mathrm{SO}_{4}$, concentrated, and purified by silica gel chromatography. Elution with cyclohexane $/ \mathrm{CH}_{2} \mathrm{Cl}_{2}(50 / 1)$ gave a yellow oil $(15 \mathrm{mg}, 5.0 \%) ;{ }^{1} \mathrm{H}-\mathrm{NMR}$ $\left(300 \mathrm{MHz} \mathrm{CDCl}_{3}\right) \delta 5.82\left(\mathrm{~m}, 1 \mathrm{H}, \mathrm{CH}=\mathrm{CH}_{2}\right), 5.16-5.10\left(\mathrm{~m}, 2 \mathrm{H}, \mathrm{CH}=\mathrm{CH}_{2}\right), 4.66(\mathrm{brd}, J=51.3 \mathrm{~Hz}, 1 \mathrm{H}$, $\mathrm{CH}-\mathrm{F}), 2.43-2.29(\mathrm{~m}, 4 \mathrm{H}), 2.15\left(\mathrm{q}, J=6.9 \mathrm{~Hz}, 2 \mathrm{H}, \mathrm{CH}_{2} \mathrm{CH}_{3}\right), 1.85-1.69(\mathrm{~m}, 2 \mathrm{H}), 1.11(\mathrm{t}, J=7.2 \mathrm{~Hz}$, $\left.3 \mathrm{H}, \mathrm{CH}_{3}\right) .{ }^{13} \mathrm{C}-\mathrm{NMR}\left(75 \mathrm{MHz}, \mathrm{CDCl}_{3}\right) \delta 133.0\left(\mathrm{~d}, \mathrm{~J}=6.0 \mathrm{~Hz}, \mathrm{CH}=\mathrm{CH}_{2}\right), 117.9\left(\mathrm{CH}=\mathrm{CH}_{2}\right), 92.0$ $(\mathrm{d}, J=168.9 \mathrm{~Hz}, \mathrm{CH}-\mathrm{F}), 82.3,78.1,39.3(\mathrm{~d}, J=21.2 \mathrm{~Hz}), 34.0(\mathrm{~d}, J=20.9 \mathrm{~Hz}), 14.7(\mathrm{~d}, J=5.2 \mathrm{~Hz}), 14.2$, 12.4. LRMS (ESI) $m / z$ calcd. for $\mathrm{C}_{10} \mathrm{H}_{16} \mathrm{~F}^{+}[\mathrm{M}+\mathrm{H}]^{+}:$155.1; found: 155.1 .

\subsection{General Precedure for Preparation of $\mathrm{Co}_{2}(\mathrm{CO})_{6}-$ Alkyne Complexes and Compound Characterization}

A mixture of alkyne 1 ( $2 \mathrm{mmol}, 1.0$ equiv. $), \mathrm{Co}_{2}(\mathrm{CO})_{8}$ (2.2 mmol, 1.1 equiv.) in $\mathrm{CH}_{2} \mathrm{Cl}_{2}(0.5 \mathrm{~mL})$ was stirred at room temperature under air atmosphere (balloon) until thin-layer chromatography (TLC) monitoring showed all alkyne consumed (about $1 \mathrm{~h}$ ). The solvent was removed under reduced pressure, and the residue was purified by silica gel column chromatography to provide $\mathrm{Co}_{2}(\mathrm{CO})_{6}$-alkyne complex 2.

Hexacarbonyl[ $\mu-\left[(7,8-\eta: 7,8-\eta)\right.$ oct-1-en-7-yn-4-ol]]-dicobalt-(Co-Co) (2a): Dark red oil, yield 57\%; ${ }^{1} \mathrm{H}-\mathrm{NMR}$ $\left(500 \mathrm{MHz}_{\mathrm{CDCl}}\right) \delta 6.02(\mathrm{~s}, 1 \mathrm{H}, \equiv \mathrm{CH}), 5.83\left(\mathrm{~m}, 1 \mathrm{H}, \mathrm{CH}=\mathrm{CH}_{2}\right), 5.19-5.15\left(\mathrm{~m}, 2 \mathrm{H}, \mathrm{CH}=\mathrm{CH}_{2}\right), 3.79(\mathrm{~m}, 1 \mathrm{H}$, $\mathrm{CH}-\mathrm{OH}), 3.09(\mathrm{~m}, 1 \mathrm{H}), 2.92(\mathrm{~m}, 1 \mathrm{H}), 2.36(\mathrm{~m}, 1 \mathrm{H}), 2.22(\mathrm{~m}, 1 \mathrm{H}), 1.80(\mathrm{~m}, 2 \mathrm{H}), 1.66(\mathrm{~d}, J=4.0 \mathrm{~Hz}, 1 \mathrm{H}$, $\mathrm{OH}) .{ }^{13} \mathrm{C}-\mathrm{NMR}\left(125 \mathrm{MHz}, \mathrm{CDCl}_{3}\right) \delta 199.9(\mathrm{br}, \mathrm{CO} \times 6), 134.2(\mathrm{C}=\mathrm{CH}), 118.8\left(\mathrm{CH}=\mathrm{CH}_{2}\right), 97.1(\mathrm{C} \equiv \mathrm{CH})$, $73.1(\mathrm{C} \equiv \mathrm{CH}), 69.9(\mathrm{CHOH}), 42.1,38.9,30.4$. HRMS (EI) $m / z$ calcd. for $\mathrm{C}_{13} \mathrm{H}_{162} \mathrm{Co}_{2} \mathrm{O}_{6}{ }^{+}\left[\mathrm{M}^{+}-\mathrm{CO}\right]$ : 381.9292; found: 381.9300 .

Hexacarbonyl[ $\mu-\left[(1,2-\eta: 1,2-\eta)\right.$ non-1-yn-5-ol]]-dicobalt-(Co-Co) (2b): Dark red oil, yield 58\%; ${ }^{1} \mathrm{H}-\mathrm{NMR}$ $\left(300 \mathrm{MHz} \mathrm{CDCl}_{3}\right) \delta 6.01(\mathrm{~s}, 1 \mathrm{H}, \equiv \mathrm{CH}), 3.92(\mathrm{~m}, 1 \mathrm{H}, \mathrm{CHOH}), 3.07(\mathrm{ddd}, J=15.6,9.6,6.3 \mathrm{~Hz}, 1 \mathrm{H}), 2.89$ $(\mathrm{ddd}, J=15.6,9.9,6.3 \mathrm{~Hz}, 1 \mathrm{H}), 1.76(\mathrm{~m}, 2 \mathrm{H}), 1.49-1.43(\mathrm{~m}, 4 \mathrm{H}), 1.38-1.33(\mathrm{~m}, 3 \mathrm{H}), 0.92(\mathrm{t}, J=6.6 \mathrm{~Hz}$, $\left.3 \mathrm{H}, \mathrm{CH}_{3}\right) \cdot{ }^{13} \mathrm{C}-\mathrm{NMR}\left(75 \mathrm{MHz}, \mathrm{CDCl}_{3}\right) \delta 200.0(\mathrm{br}, \mathrm{CO} \times 6), 97.3(\mathrm{C} \equiv \mathrm{CH}), 73.0(\mathrm{C} \equiv \mathrm{CH}), 71.3(\mathrm{CHOH})$, 39.5, 37.2, 30.3, 27.7, 22.7, $14.0\left(\mathrm{CH}_{3}\right)$. HRMS (EI) $\mathrm{m} / \mathrm{z}$ calcd. for $\mathrm{C}_{14} \mathrm{H}_{16} \mathrm{Co}_{2} \mathrm{O}_{6}{ }^{+}\left[\mathrm{M}^{+}-\mathrm{CO}\right]$ : 397.9605; found: 397.9615 .

Hexacarbonyl[ $\mu-\left[(4,5-\eta: 4,5-\eta)\right.$ pent-4-yn-2-ol]]-dicobalt-(Co-Co) (2c): Dark red oil, yield 67\%; ${ }^{1} \mathrm{H}-\mathrm{NMR}$ $\left(300 \mathrm{MHz} \mathrm{CDCl}_{3}\right) \delta 6.10(\mathrm{~s}, 1 \mathrm{H}, \equiv \mathrm{CH}), 3.95(\mathrm{~m}, 1 \mathrm{H}, \mathrm{CHOH}), 3.01\left(\mathrm{~d}, J=4.8 \mathrm{~Hz}, 2 \mathrm{H}, \mathrm{CH}_{2}\right), 1.57$ $(\mathrm{d}, J=6.3 \mathrm{~Hz}, 1 \mathrm{H}, \mathrm{OH}), 1.35\left(\mathrm{~d}, J=5.4 \mathrm{~Hz}, 3 \mathrm{H}, \mathrm{CH}_{3}\right) .{ }^{13} \mathrm{C}-\mathrm{NMR}\left(75 \mathrm{MHz}, \mathrm{CDCl}_{3}\right) \delta 199.8(\mathrm{br}, \mathrm{CO} \times 6)$, $92.0(\mathrm{C} \equiv \mathrm{CH}), 73.9(\mathrm{C} \equiv \mathrm{CH}), 68.7(\mathrm{CHOH}), 43.7\left(\mathrm{CH}_{2}\right), 23.7\left(\mathrm{CH}_{3}\right)$. HRMS (EI) $m / z$ calcd. for $\mathrm{C}_{10} \mathrm{H}_{8} \mathrm{Co}_{2} \mathrm{O}_{6}{ }^{+}\left[\mathrm{M}^{+}-\mathrm{CO}\right]$ : 341.8979; found: 341.8987.

Hexacarbonyl[ $\mu-[(5,6-\eta: 5,6-\eta) h e x-5-y n-3-o l]]-d i c o b a l t-(C o-C o)(2 d)$ : Dark red oil, yield 78\%; ${ }^{1} \mathrm{H}-\mathrm{NMR}$ $\left(300 \mathrm{MHz}, \mathrm{CDCl}_{3}\right) \delta 6.09(\mathrm{~s}, 1 \mathrm{H}, \equiv \mathrm{CH}), 3.66(\mathrm{~m}, 1 \mathrm{H}, \mathrm{CHOH}), 3.00\left(\mathrm{~m}, 2 \mathrm{H}, \equiv \mathrm{CCH}_{2}\right), 1.76(\mathrm{~d}, J=3.3 \mathrm{~Hz}$, 
1H, OH), $1.63\left(\mathrm{~m}, 2 \mathrm{H}, \mathrm{CH}_{2} \mathrm{CH}_{3}\right), 1.01\left(\mathrm{t}, J=7.2 \mathrm{~Hz}, 3 \mathrm{H}, \mathrm{CH}_{3}\right) .{ }^{13} \mathrm{C}-\mathrm{NMR}\left(75 \mathrm{MHz}, \mathrm{CDCl}_{3}\right) \delta 199.8$ (br, $\mathrm{CO} \times 6), 92.5(\mathrm{C} \equiv \mathrm{CH}), 74.1(\mathrm{C} \equiv \mathrm{CH}), 73.9(\mathrm{CHOH}), 41.5\left(\equiv \mathrm{CCH}_{2}\right), 30.2\left(\mathrm{CH}_{2} \mathrm{CH}_{3}\right), 9.7\left(\mathrm{CH}_{3}\right)$. HRMS (EI) $m / z$ calcd. for $\mathrm{C}_{11} \mathrm{H}_{10} \mathrm{Co}_{2} \mathrm{O}_{6}{ }^{+}\left[\mathrm{M}^{+}-\mathrm{CO}\right.$ ]: 355.9136; found: 355.9142 .

Hexacarbonyl[ $\mu-\left[(1,2-\eta: 1,2-\eta)\right.$ pent-1-yn-3-ol]]-dicobalt-(Co-Co) (2e): Dark red oil, yield $84 \% ;{ }^{1} \mathrm{H}-\mathrm{NMR}$ $\left(600 \mathrm{MHz}, \mathrm{CDCl}_{3}\right) \delta 6.06(\mathrm{~d}, J=0.6 \mathrm{~Hz}, 1 \mathrm{H}, \equiv \mathrm{CH}), 4.63(\mathrm{ddd}, J=7.8,5.4,5.4 \mathrm{~Hz}, 1 \mathrm{H}, \mathrm{CHOH}), 1.84$ $(\mathrm{d}, J=5.4 \mathrm{~Hz}, 1 \mathrm{H}, \mathrm{OH}), 1.76\left(\mathrm{~m}, 1 \mathrm{H}\right.$ of $\left.\mathrm{CH}_{2}\right), 1.70\left(\mathrm{~m}, 1 \mathrm{H}\right.$ of $\left.\mathrm{CH}_{2}\right), 1.09\left(\mathrm{t}, J=7.2 \mathrm{~Hz}, 3 \mathrm{H}, \mathrm{CH}_{3}\right) .{ }^{13} \mathrm{C}-\mathrm{NMR}$ $\left(150 \mathrm{MHz}, \mathrm{CDCl}_{3}\right) \delta 199.6(\mathrm{br}, \mathrm{CO} \times 6), 99.8(\mathrm{C} \equiv \mathrm{CH}), 73.9,71.6,32.9\left(\mathrm{CH}_{2}\right), 10.6\left(\mathrm{CH}_{3}\right)$. HRMS (EI) $\mathrm{m} / z$ calcd. for $\mathrm{C}_{10} \mathrm{H}_{8} \mathrm{Co}_{2} \mathrm{O}_{6}{ }^{+}\left[\mathrm{M}^{+}\right.$]: 369.8929 ; found: 369.8933 .

Hexacarbonyl[ $\mu-[(1,2-\eta: 1,2-\eta)-3-m e t h y l p e n t-1-y n-3-o l]]-d i c o b a l t-\left(C_{0}-C_{0}\right)(2 \mathbf{f})$ : Dark red oil, yield 78\%; ${ }^{1} \mathrm{H}-\mathrm{NMR}\left(300 \mathrm{MHz}, \mathrm{CDCl}_{3}\right) \delta 6.06(\mathrm{~s}, 1 \mathrm{H}, \equiv \mathrm{CH}), 1.78\left(\mathrm{~m}, 2 \mathrm{H}, \mathrm{CH}_{2}\right), 1.73(\mathrm{~s}, 1 \mathrm{H}, \mathrm{OH}), 1.49\left(\mathrm{~s}, 3 \mathrm{H}, \mathrm{CH}_{3}\right)$, $1.03\left(\mathrm{t}, J=7.2 \mathrm{~Hz}, 3 \mathrm{H}, \mathrm{CH}_{2} \mathrm{CH}_{3}\right) .{ }^{13} \mathrm{C}-\mathrm{NMR}\left(75 \mathrm{MHz}, \mathrm{CDCl}_{3}\right) \delta 199.6(\mathrm{br}, \mathrm{CO} \times 6), 105.2(\mathrm{C} \equiv \mathrm{CH}), 74.9$, 72.1, 37.8 $\left(\mathrm{CH}_{2}\right), 29.8\left(\mathrm{CH}_{3}\right), 8.7\left(\mathrm{CH}_{2} \mathrm{CH}_{3}\right)$. HRMS (EI) $\mathrm{m} / z$ calcd. for $\mathrm{C}_{12} \mathrm{H}_{10} \mathrm{Co}_{2} \mathrm{O}_{7}^{+}\left[\mathrm{M}^{+}\right]: 383.9085$; found: 383.9090 .

Hexacarbonyl[ $\mu-\left[(7,8-\eta: 7,8-\eta)\right.$ dec-1-en-7-yn-4-ol]]-dicobalt-(Co-Co) (2g): Dark red oil, yield 69\%; ${ }^{1} \mathrm{H}-\mathrm{NMR}$ $\left(300 \mathrm{MHz}, \mathrm{CDCl}_{3}\right) \delta 5.82\left(\mathrm{~m}, 1 \mathrm{H}, \mathrm{CH}=\mathrm{CH}_{2}\right), 5.20-5.15\left(\mathrm{~m}, 2 \mathrm{H}, \mathrm{CH}=\mathrm{CH}_{2}\right), 3.79(\mathrm{brd}, J=3.9 \mathrm{~Hz}, 1 \mathrm{H}$, $\mathrm{CH}-\mathrm{OH}), 3.08(\mathrm{~m}, 1 \mathrm{H}), 2.91(\mathrm{~m}, 1 \mathrm{H}), 2.86\left(\mathrm{q}, J=7.2 \mathrm{~Hz}, 2 \mathrm{H}, \mathrm{CH}_{2} \mathrm{CH}_{3}\right), 2.36(\mathrm{~m}, 1 \mathrm{H}), 2.22(\mathrm{~m}, 1 \mathrm{H}), 1.82$ $(\mathrm{m}, 2 \mathrm{H}), 1.69(\mathrm{~d}, J=3.9 \mathrm{~Hz}, 1 \mathrm{H}, \mathrm{OH}), 1.29\left(\mathrm{t}, J=7.2 \mathrm{~Hz}, 3 \mathrm{H}, \mathrm{CH}_{3}\right) .{ }^{13} \mathrm{C}-\mathrm{NMR}\left(75 \mathrm{MHz}, \mathrm{CDCl}_{3}\right) \delta 200.3$ $(\mathrm{br}, \mathrm{CO} \times 6), 134.2\left(\mathrm{CH}=\mathrm{CH}_{2}\right), 118.8\left(\mathrm{CH}=\mathrm{CH}_{2}\right), 101.8,99.0,70.0(\mathrm{CH}-\mathrm{OH}), 42.0,38.3,30.1,27.0,15.6$ $\left(\mathrm{CH}_{3}\right)$. HRMS (EI) $\mathrm{m} / z$ calcd. for $\mathrm{C}_{15} \mathrm{H}_{16} \mathrm{Co}_{2} \mathrm{O}_{6}{ }^{+}\left[\mathrm{M}^{+}-\mathrm{CO}\right.$ ]: 409.9605 ; found: 409.9607.

Hexacarbonyl[ $\mu-[(7,8-\eta: 7,8-\eta)-8-($ trimethylsilyl)oct-1-en-7-yn-4-ol]]-dicobalt-(Co-Co) (2h): Dark red oil, yield $49 \% ;{ }^{1} \mathrm{H}-\mathrm{NMR}\left(300 \mathrm{MHz}, \mathrm{CDCl}_{3}\right) \delta 5.82\left(\mathrm{~m}, 1 \mathrm{H}, \mathrm{CH}=\mathrm{CH}_{2}\right), 5.21-5.16\left(\mathrm{~m}, 2 \mathrm{H}, \mathrm{CH}=\mathrm{CH}_{2}\right), 3.80$ $(\mathrm{m}, 1 \mathrm{H}, \mathrm{CH}-\mathrm{OH}), 3.20(\mathrm{~m}, 1 \mathrm{H}), 2.96(\mathrm{~m}, 1 \mathrm{H}), 2.40(\mathrm{~m}, 1 \mathrm{H}), 2.23(\mathrm{~m}, 1 \mathrm{H}), 1.82(\mathrm{~m}, 2 \mathrm{H}), 1.68(\mathrm{~s}, 1 \mathrm{H}$, $\mathrm{OH}), 0.30\left(\mathrm{~s}, 9 \mathrm{H}, \mathrm{CH}_{3} \times 3\right) .{ }^{13} \mathrm{C}-\mathrm{NMR}\left(75 \mathrm{MHz}, \mathrm{CDCl}_{3}\right) \delta 200.6(\mathrm{br}, \mathrm{CO} \times 6), 134.1\left(\mathrm{CH}=\mathrm{CH}_{2}\right), 118.8$ $\left(\mathrm{CH}=\mathrm{CH}_{2}\right), 112.3(\equiv \mathrm{C}-\mathrm{Si}), 79.1(\mathrm{C} \equiv \mathrm{C}-\mathrm{Si}), 70.0(\mathrm{CH}-\mathrm{OH}), 42.0,39.2,31.4,0.65\left(\mathrm{SiCH}_{3} \times 3\right)$. HRMS (EI) $m / z$ calcd. for $\mathrm{C}_{16} \mathrm{H}_{20} \mathrm{Co}_{2} \mathrm{O}_{6} \mathrm{Si}^{+}$[M ${ }^{+}-\mathrm{CO}$ ]: 453.9688; found: 453.9693.

Hexacarbonyl $[\mu-[(7,8-\eta: 7,8-\eta)-4$-chlorooct-1-en-7-yne]]-dicobalt-(Co-Co) (2i): Dark red oil, yield 74\%; ${ }^{1} \mathrm{H}-\mathrm{NMR}\left(300 \mathrm{MHz}, \mathrm{CDCl}_{3}\right) \delta 6.03(\mathrm{~s}, 1 \mathrm{H}, \equiv \mathrm{CH}), 5.85\left(\mathrm{~m}, 1 \mathrm{H}, \mathrm{CH}=\mathrm{CH}_{2}\right), 5.18-5.13\left(\mathrm{~m}, 2 \mathrm{H}, \mathrm{CH}=\mathrm{CH}_{2}\right)$, $4.05(\mathrm{~m}, 1 \mathrm{H}, \mathrm{CH}-\mathrm{Cl}), 3.11(\mathrm{~m}, 1 \mathrm{H}), 2.97(\mathrm{~m}, 1 \mathrm{H}), 2.57(\mathrm{~m}, 2 \mathrm{H}), 2.05(\mathrm{~m}, 2 \mathrm{H}) .{ }^{13} \mathrm{C}-\mathrm{NMR}\left(75 \mathrm{MHz}, \mathrm{CDCl}_{3}\right)$ $\delta 199.9(\mathrm{br}, \mathrm{CO} \times 6), 133.6\left(\mathrm{CH}=\mathrm{CH}_{2}\right), 118.4\left(\mathrm{CH}=\mathrm{CH}_{2}\right), 95.7(\mathrm{C} \equiv \mathrm{CH}), 73.1(\mathrm{C} \equiv \mathrm{CH}), 61.2(\mathrm{CH}-\mathrm{Cl}), 42.7$, 39.5, 30.8. HRMS (EI) $m / z$ calcd. for $\mathrm{C}_{13} \mathrm{H}_{11} \mathrm{Co}_{2}{ }^{35} \mathrm{ClO}_{5}{ }^{+}\left[\mathrm{M}^{+}-\mathrm{CO}\right]: 399.8954$; found: 399.8960 .

Hexacarbonyl[ $\mu-[(7,8-\eta: 7,8-\eta)-4-$ fluorodec-1-en-7-yne]]-dicobalt-(Co-Co) (2j): Dark red oil, yield 90\%; ${ }^{1} \mathrm{H}-\mathrm{NMR}\left(600 \mathrm{MHz}, \mathrm{CDCl}_{3}\right) \delta 5.84\left(\mathrm{ddt}, J=24.6,17.4,7.2 \mathrm{~Hz}, 1 \mathrm{H}, \mathrm{CH}=\mathrm{CH}_{2}\right), 5.17-5.13(\mathrm{~m}, 2 \mathrm{H}$, $\left.\mathrm{CH}=\mathrm{CH}_{2}\right), 4.66(\mathrm{brd}, J=48.6 \mathrm{~Hz}, 1 \mathrm{H}, \mathrm{CH}-\mathrm{F}), 3.06(\mathrm{~m}, 1 \mathrm{H}), 2.91(\mathrm{~m}, 1 \mathrm{H}), 2.86(\mathrm{q}, J=7.2 \mathrm{~Hz}, 2 \mathrm{H}$, $\left.\mathrm{CH}_{2} \mathrm{CH}_{3}\right), 2.46(\mathrm{~m}, 2 \mathrm{H}), 1.93(\mathrm{~m}, 2 \mathrm{H}), 1.29\left(\mathrm{t}, J=7.2 \mathrm{~Hz}, 3 \mathrm{H}, \mathrm{CH}_{3}\right) .{ }^{13} \mathrm{C}-\mathrm{NMR}\left(150 \mathrm{MHz}, \mathrm{CDCl}_{3}\right) \delta$ $200.1(\mathrm{br}, \mathrm{CO} \times 6), 132.6\left(\mathrm{~d}, J=6.3 \mathrm{~Hz}, \mathrm{CH}=\mathrm{CH}_{2}\right), 118.3\left(\mathrm{CH}=\mathrm{CH}_{2}\right), 101.8(\mathrm{C} \equiv \mathrm{CEt}), 98.2(\mathrm{C} \equiv \mathrm{CEt}), 92.5$ $(\mathrm{d}, J=170.1 \mathrm{~Hz}, \mathrm{CH}-\mathrm{F}), 39.4\left(\mathrm{~d}, J=21.3 \mathrm{~Hz}, \mathrm{CH}_{2} \mathrm{CH}=\mathrm{CH}_{2}\right), 36.2\left(\mathrm{~d}, J=20.7 \mathrm{~Hz}, \mathrm{CH}_{2} \mathrm{CH}_{2} \mathrm{C} \equiv \mathrm{C}\right), 29.3$ $\left(\mathrm{d}, J=4.1 \mathrm{~Hz}, \mathrm{C} \equiv \mathrm{CCH}_{2} \mathrm{CH}_{2}\right), 27.0\left(\mathrm{CH}_{2} \mathrm{CH}_{3}\right), 15.6\left(\mathrm{CH}_{3}\right)$. HRMS (EI) $\mathrm{m} / z$ calcd. for $\mathrm{C}_{15} \mathrm{H}_{15} \mathrm{Co}_{2} \mathrm{FO}_{5}{ }^{+}$ [M+ $-\mathrm{CO}]:$ 411.9562; found: 411.9570.

Hexacarbonyl[ $\mu-[2-((3,4-\eta: 3,4-\eta)$ but-3-yn-1-yloxy)tetrahydro-2H-pyran]]-dicobalt-(Co-Co) (2k): Dark red oil, yield 65\%; ${ }^{1} \mathrm{H}-\mathrm{NMR}\left(500 \mathrm{MHz}, \mathrm{CDCl}_{3}\right) \delta 6.03(\mathrm{~s}, 1 \mathrm{H}, \equiv \mathrm{CH}), 4.63(\mathrm{t}, J=4.0 \mathrm{~Hz}, 1 \mathrm{H}, \mathrm{O}-\mathrm{CH}-\mathrm{O}), 3.99$ $(\mathrm{m}, 1 \mathrm{H}), 3.88(\mathrm{~m}, 1 \mathrm{H}), 3.60(\mathrm{~m}, 1 \mathrm{H}), 3.53(\mathrm{~m}, 1 \mathrm{H}), 3.15(\mathrm{~m}, 2 \mathrm{H}), 1.84(\mathrm{~m}, 1 \mathrm{H}), 1.72(\mathrm{~m}, 1 \mathrm{H}), 1.62-1.51$ $(\mathrm{m}, 4 \mathrm{H}) .{ }^{13} \mathrm{C}-\mathrm{NMR}\left(125 \mathrm{MHz}, \mathrm{CDCl}_{3}\right) \delta 199.9(\mathrm{br}, \mathrm{CO} \times 6), 98.9(\mathrm{O}-\mathrm{CH}-\mathrm{O}), 92.9(\mathrm{C} \equiv \mathrm{CH}), 73.7(\mathrm{C} \equiv \mathrm{CH})$, 67.3, 62.4, 34.1, 30.6, 25.4, 19.5. HRMS (EI) $m / z$ calcd. for $\mathrm{C}_{14} \mathrm{H}_{14} \mathrm{Co}_{2} \mathrm{O}_{7}{ }^{+}\left[\mathrm{M}^{+}-\mathrm{CO}\right]: 411.9398$; found: 411.9400 . 


\section{Conclusions}

We have developed a simple and general method to separate the enantiomers of chiral alkynes using their in situ generated cobalt-complex by chiral column HPLC. HPLC analysis of enantiomeric purity of metal complexes with carbon-metal bonds is not usual [16]. Our method is particularly useful for the alkynes without chromophores and other derivable groups. Since the decomplexation of Co-alkyne complexes is well known [17], our method is also promisingly suitable for the preparative resolution of racemic alkynes.

Supplementary Materials: Supplementary materials are available online. (1) General Information; (2) UV-Vis spectra of $\mathbf{1 a}$ and $\mathbf{2 a}$; (3) ${ }^{1} \mathrm{H}$ - and ${ }^{13} \mathrm{C}-\mathrm{NMR}$ spectra of $\mathbf{1} \mathbf{b}$, and $\mathbf{1} \mathbf{g}-\mathbf{j}$; (4) HPLC chart of $\mathbf{1 a}$; (5) ${ }^{1} \mathrm{H}-{ }^{13} \mathrm{C}-\mathrm{NMR}$ spectra and HPLC charts of $\mathbf{2 a - k}$; (6) HPLC monitor of the reaction of $\mathbf{1 a}$ and $\mathrm{Co}_{2}(\mathrm{CO})_{8}$.

Acknowledgments: This work was financially supported by the National Natural Science Foundation of China (21502234 and 21602256), Beijing Natural Science Foundation (2164074), Natural Science Foundation of Shanxi (2013011011-2), Research Funds from State Key Laboratory of Bioactive Substance and Function of Natural Medicines (GTZB201404), CAMS Innovation Fund for Medical Sciences (CIFMS, 2016-I2M-3-009), and Fundamental Research Funds for CAMS/PUMC (2016RC350004). We thank Li Li, IMM, CAMS/PUMC for the useful discussion and Leilei Zhang, IMM, CAMS/PUMC for high resolution mass spectrometric assistance.

Author Contributions: X.L. and S.X. conceived the experiments; X.L., Y.G. and S.X. designed the experiments; J.W., X.L. and Y.G. performed the experiments; Q.L., J.L., Y.G., S.L., X.W. and S.X. analyzed the data; Q.L., J.L., X.W. and S.X. wrote the paper.

Conflicts of Interest: The authors declare no conflict of interest.

\section{References}

1. Trost, B.M.; Li, C.-J. Modern Alkyne Chemistry: Catalytic and Atom-Economic Transformations, 1st ed.; Wiley-VCH Verlag GmbH \& Co. KGaA: Weinheim, Germany, 2015.

2. Trost, B.M.; Masters, J.T. Transition metal-catalyzed couplings of alkynes to 1,3-enynes: Modern methods and synthetic applications. Chem. Soc. Rev. 2016, 45, 2212-2238. [CrossRef] [PubMed]

3. Yang, Z.; Shi, L.L. Exploring the complexity-generating features of the Pauson-Khand reaction from a synthetic perspective. Eur. J. Org. Chem. 2016, 14, 2356-2368.

4. Kacprzak, K.; Skiera, I.; Piasecka, M.; Paryzek, Z. Alkaloids and isoprenoids modification by copper(I)-catalyzed Huisgen 1,3-dipolar cycloaddition (click chemistry): Toward new functions and molecular architectures. Chem. Rev. 2016, 116, 2767-2770. [CrossRef] [PubMed]

5. Nagle, D.G.; Geralds, R.-S.; Yoo, H.-D.; Gerwick, W.H.; Kim, T.-S.; Nambu, M.; White, J.D. Absolute configuration of curacin A, a novel antimitotic agent from the tropical marine cyanobacterium Lyngbya majuscula. Tetrahedron Lett. 1995, 36, 1189-1192. [CrossRef]

6. White, J.D.; Kim, T.-S.; Nambu, M. Synthesis of curacin A: A powerful antimitotic from the cyanobacterium Lyngbya majuscule. J. Am. Chem. Soc. 1995, 117, 5612-5613. [CrossRef]

7. White, J.D.; Kim, T.-S.; Nambu, M. Absolute configuration and total synthesis of (+)-curacin A, an antiproliferative agent from the cyanobacterium Lyngbya majuscula. J. Am. Chem. Soc. 1997, 119, 103-111. [CrossRef]

8. Codesido, E.M.; Cid, M.M.; Castedo, L.; Mouriño, A.; Granja, J.R. Synthesis of vitamin D analogues with a 2-hydroxy-3-deoxy ring A. Tetrahedron Lett. 2000, 41, 5861-5864. [CrossRef]

9. García-Fandiño, R.; Aldegunde, M.J.; Codesido, E.M.; Castedo, L.; Granja, J.R. RCM for the construction of novel steroid-like polycyclic systems. 1 . Studies on the Synthesis of a $\mathrm{PreD}_{3}-\mathrm{D}_{3}$ transition state analogue. J. Org. Chem. 2005, 70, 8281-8290. [CrossRef] [PubMed]

10. Nicolaou, K.C.; Leung, G.Y.C.; Dethe, D.H.; Guduru, R.; Sun, Y.-P.; Lim, C.S.; Chen, D.Y.-K. Chemical synthesis and biological evaluation of palmerolide A analogues. J. Am. Chem. Soc. 2008, 130, 10019-10023. [CrossRef] [PubMed]

11. Ngai, M.H.; Yang, P.-Y.; Liu, K.; Shen, Y.; Wenk, M.R.; Yao, S.Q.; Lear, M.J. Click-based synthesis and proteomic profiling of lipstatin analogues. Chem. Commun. 2010, 46, 8335-8337. [CrossRef] [PubMed]

12. Shibata, H.; Tsuchikawa, H.; Hayashi, T.; Matsumori, N.; Murata, M.; Usui, T. Modification of bafilomycin structure to efficiently synthesize solid-state NMR probes that selectively bind to vacuolar-type ATPase. Chem. Asian J. 2015, 10, 915-924. [CrossRef] [PubMed] 
13. Iseki, K.; Mizuno, S.; Kuroki, Y.; Kobayashi, Y. A chiral formamide: Design and application to catalytic asymmetric synthesis. Tetrahedron Lett. 1998, 39, 2767-2770. [CrossRef]

14. Seco, J.M.; Quinoa, E.; Riguera, R. The assignment of absolute configuration by NMR. Chem. Rev. 2004, 104, 17-118. [CrossRef]

15. Miyamoto, H.; Hirano, T.; Okawa, Y.; Nakazaki, A.; Kobayashi, S. Stereoselective synthesis of spirocyclic oxindoles based on a one-pot Ullmann coupling/Claisen rearrangement and its application to the synthesis of a hexahydropyrrolo[2,3-b] indole alkaloid. Tetrahedron 2013, 69, 9481-9493. [CrossRef]

16. Wang, P.; Lee, H.K. Recent applications of high-performance liquid chromatography to the analysis of metal complexes. J. Chromatogr. A 1997, 789, 437-451. [CrossRef]

17. Nicholas, K.M.; Pettit, R. An alkyne protecting group. Tetrahedron Lett. 1971, 12, 9481-9493. [CrossRef]

Sample Availability: Samples of the compounds $\mathbf{2 a - k}$ are available from the authors.

(C) 2017 by the authors. Licensee MDPI, Basel, Switzerland. This article is an open access article distributed under the terms and conditions of the Creative Commons Attribution (CC BY) license (http:/ / creativecommons.org/licenses/by/4.0/). 